1 Running head: UNDER-RESOURCED OR OVERLOADED?

2

3

4

5

6 7

8

9

10

11

12

13

14

15

16

17 International Centre for Language and Communicative Development (LuCiD)

18

19

20

21

22

23

24 Department of Psychology, Lancaster University, Lancaster, United Kingdom, LA1 4YF.

25 Email: sam.jones@lancaster.ac.uk. Telephone: +44 (0) 1524593698.

\section{language disorder}

Samuel David Jones and Gert Westermann

Department of Psychology, Lancaster University

8758 words

Author note

This work was supported by Economic and Social Research Council (ESRC)

[ES/S007113/1 and ES/L008955/1]. We have no conflicts of interest to disclose. A preprint

of this manuscript was posted on the Open Science Framework on 25 ${ }^{\text {th }}$ May 2021

(https://osf.io/rb5jf). The theoretical view described in this manuscript was presented at the

Biennial Meeting of the Society for Research in Child Development 2021 and the ESRC

LuCiD Conference 2021.

Correspondence concerning this article should be addressed to Sam Jones, 


\section{Abstract}

27 Dominant theoretical accounts of developmental language disorder (DLD) commonly invoke working memory capacity limitations. In the current report, we present an alternative view: That working memory in DLD is not under-resourced but overloaded due to operating on speech representations with low discriminability. This account is developed through computational simulations involving deep convolutional neural networks trained on spoken word spectrograms in which information is either retained to mimic typical development or degraded to mimic the auditory processing deficits identified among some children with DLD. We assess not only spoken word recognition accuracy and predictive probability and entropy (i.e., predictive distribution spread), but also use mean-field-theory based manifold analysis to assess; (i) internal speech representation dimensionality, and (ii) classification capacity, a measure of the networks' ability to isolate any given internal speech representation that is used as a proxy for attentional control. We show that instantiating a low-level auditory processing deficit results in the formation of internal speech representations with atypically high dimensionality, and that classification capacity is exhausted due to low representation separability. These representation and control deficits underpin not only lower performance accuracy but also greater uncertainty even when making accurate predictions in a simulated spoken word recognition task (i.e., predictive distributions with low maximum probability and high entropy), which replicates the response delays and word finding difficulties often seen in DLD. Overall, these simulations demonstrate a theoretical account of speech representation and processing deficits in DLD in which working memory capacity limitations play no causal role. Keywords: developmental language disorder, spoken word recognition, word learning, convolutional neural network, manifold geometry 
Under-resourced or overloaded? Rethinking working memory deficits in developmental

\section{language disorder}

Learning language is a central aspect of child development and is often mastered with astonishing ease despite the complexity of language and a lack of direct instruction.

Nevertheless, not all children succeed equally in acquiring language. In developmental language disorder (DLD), deficits in spoken language comprehension and production severe enough to affect the child's wellbeing are observed despite no obvious biomedical cause (Bishop et al., 2016). Although DLD is widespread, affecting approximately $7.5 \%$ of English-speaking children (Norbury et al., 2016), much remains unknown about the causal mechanisms underlying this condition.

A dominant feature of existing causal accounts of DLD is an emphasis on the role of working memory. Apparently uniformly, research in this area has taken lead from Baddeley and Hitch's (1974) multi-component model, which comprises a central executive that attends to and manipulates information stored temporarily in one of three modality-specific buffer systems; the visuospatial sketchpad, the episodic buffer, and the phonological loop. Research into the causal origins of DLD has focused principally on the role of the phonological loop in the temporary retention of speech signals, and the role of the central executive in retrieving and manipulating speech signals. ${ }^{1}$ Performance deficits in tasks thought to test the integrity of the working memory system are perhaps the most consistent finding in DLD research. Children with DLD commonly score poorly, for instance, in the non-word repetition task, in which participants are required to repeat recently heard auditory stimuli such as doppelate, hampent, or

\footnotetext{
${ }^{1}$ As Vance (2008) has commented, the terms working memory and short-term memory are used differently and sometimes interchangeably across studies of DLD. In the current study, working memory refers to both the system supporting the temporary retention of activated long-term representations in response to a perceived stimulus (short-term memory, or the phonological loop) and the attention system that operates on (i.e., selects, inhibits, or manipulates) those activated representations (attentional control, or the central executive).
} 
glistering, a task commonly held to tap phonological loop capacity (see Vance, 2008, for review). Performance deficits in the non-word repetition task and related paradigms among children with DLD underpin the consensus view that capacity limitations in both the central executive and phonological loop subsystems of working memory play a causal role in these children's language difficulties, directly obstructing the temporary retention, retrieval, and manipulation of speech signals, and resulting in degraded long-term speech representations during learning (Archibald \& Gathercole, 2006a; Archibald \& Harder Griebeling, 2016; Delage \& Durrleman, 2018; Delage \& Frauenfelder, 2020; Durrleman \& Delage, 2016; Ellis Weismer et al., 2017; Jakubowicz, 2011; Montgomery, 1995, 2003; Zebib et al., 2020; Montgomery et al., 2019; cf. Howard \& Lely, 1995; Van Der Lely \& Howard, 1993; see also Kail, 1994, for an account citing generalized slowing rather than specific working memory capacity deficits).

Yet, despite the dominance of the causal view of working memory capacity limitations in DLD, much of the evidence cited in support of this position is correlational. A child might show a non-word repetition task performance deficit alongside a deficit in vocabulary size or sentence comprehension, for instance, and a causal association between a hypothesised underlying working memory capacity limitation and relatively poor language skills is inferred on this basis (e.g., Montgomery, 1995; note that more recent studies assess such correlations using more advanced methods, including mediation and cross-lagged designs, e.g., Blom \& Boerma, 2020). Alternatively, some studies have sought to identify domain general working memory capacity deficits in children with DLD, for instance deficits implicating both verbal and visual working memory subsystems; the former measured using tasks such as non-word repetition and the latter measured using visual pattern recognition and spatial span tasks (Archibald \& Gathercole, 2006b; Bavin et al., 2005; Henry \& Botting, 2017). Here, the identification of domain general deficits is argued to bolster the view that 
97 working memory capacity limitations play a primary role in language impairment, ensuring that performance deficits are not simply an epiphenomenon of shortfalls in long-term language knowledge. However, this position remains contentious, with some studies reporting no evidence of visual working memory task performance deficits among children

101 affected by DLD, a finding lending apparent support to the claim that the underlying problem

102 is specific to the verbal working memory system (Archibald \& Gathercole, 2006b). Seemingly stronger evidence for a causal association between working memory capacity limitations and language impairment comes from studies reporting non-word repetition task performance deficits in individuals whose language problems have been

106 resolved through intervention (Bishop et al., 1996). This pattern would apparently not be 107 expected if working memory task performance deficits purely reflected insufficient long-term 108 language knowledge. Yet, as these authors acknowledge, alongside others (e.g., Coady \& 109 Evans, 2008; Melby-Lervåg et al., 2012), the once common interpretation of non-word

110 repetition task performance as a relatively pure measure of working memory capacity,

111 specifically phonological loop capacity, is misplaced, as non-word repetition implicates a

112 wide range of skills including auditory perception, speech planning, and articulation. While

113 this more nuanced interpretation of what is measured in the non-word repetition task and

114 closely related paradigms in no way challenges the validity of using such measures to

115 identify individuals with existing language impairment, or potentially with a history of

116 language impairment, it does undermine the view that what we are detecting in administering

117 such tasks is a pure working memory capacity limitation. The picture is complex, and deficits

118 in, for instance, non-word repetition task performance despite largely resolved language

119 difficulties may reflect residual deficits in any number of skills.

120 In our view, the causal account of working memory capacity limitations in DLD

121 remains dominant because the field lacks a cohesive alternative. This has important practical 
implications. An alternative theoretical framework in which working memory capacity limitations do not feature may not only provide a more compelling explanation of the behavioural data at hand, but it may also entail different approaches to language support. Evidence interpreted as signaling a causal association between limited working memory capacity and language deficits has motivated the development of commercial packages claiming to improve working memory capacity and in doing so boost language and educational outcomes (e.g. Jungle Memory; Alloway et al., 2013). However, if working memory capacity limitations are not a major underlying cause of language deficits then interventions may need to focus on a different aspect of cognition or language processing in order to achieve substantial and lasting effects. It is important to re-iterate that working memory task performance remains one of the best predictors of language impairment (Bishop et al., 1996; Girbau, 2016; Kalnak et al., 2014), and that the validity of using such paradigms to statistically identify individuals at risk of language problems is not in question. What is in question, is whether apparent working memory capacity limitations are the cause, rather than consequence, of the language learning and processing difficulties seen among children with

137 DLD.

\section{Rethinking working memory capacity deficits in DLD}

The view developed in this report is that working memory capacity limitations are the consequence rather than cause of children's language difficulties. Crucial to this account is

141 the notion of a capacity and performance trade-off. It is uncontroversial that long-term

142 knowledge affects working memory task performance (Vance, 2008). In both typically and atypically developing populations, performance is seen to decline (e.g., in terms of the length

144 of speech segments that can be accurately recalled) when individuals are presented with unfamiliar stimuli, as seen in word-likeness effects (i.e. phonologically anomalous non-words are harder to repeat; Gathercole, 1995; Van Bon \& Van Der Pijl, 1997) and in responses to 
147 noisy stimuli (Marrone et al., 2015). The idea of a capacity and performance trade-off

148 suggests that this drop in performance emerges due to working memory being overloaded as

149 a result of heightened processing demands. In contrast, faced with broadly familiar, non-

150 noisy stimuli, processing resources are not under pressure and so more information can be

151 retained.

152 One possibility, then, is that performance deficits widely attributed to working memory capacity limitations among children with DLD instead reflect heightened processing

154 demands resulting from deficits in long-term language knowledge, including poorly

155 configured long-term speech representations (Kan \& Windsor, 2010). This issue may be

156 masked by the fact that the stimuli presented to children with and without DLD in working

157 memory tasks are usually matched; for example, stimuli are either all clean or all noisy across

158 groups. Yet, if a child with DLD has deficient speech encoding ability then their experience

159 of any given stimulus will be very different to that of a same-age child without language

160 impairment, increasing processing demands for this child and exhausting cognitive resources

161 that could be allocated to storage capacity. Rather than fixed, group-level disparities in

162 working memory capacity, then, the difference between children with and without DLD may

163 resemble the ostensible capacity discrepancies that can be seen in a single typically

164 developing child who is presented with noisy and then clean stimuli, and who retains more

165 information in the second instance. Children with DLD may not be under-resourced in terms

166 of their working memory capacity as the consensus holds but may instead be overloaded by

167 heightened processing demands given poorly configured long-term speech representations.

168 Though relatively unexplored, limited evidence in support of this position includes an

169 apparent absence of working memory task performance deficits between children with DLD

170 and control children matched on long-term language knowledge (Van Der Lely \& Howard,

171 1993). 
This view of working memory capacity limitations as the consequence rather than cause of language difficulties aligns well with contemporary working memory frameworks that seek to de-emphasise the role of functionally discrete, modality-specific buffers, such as the phonological loop, in favour of a relatively parsimonious characterization of working memory in terms of activated long-term memory plus attention (Adams et al., 2018; Chai et al., 2018; Cowan, 1995; D’Esposito \& Postle, 2015; McElree, 2006; Oberauer, 2013, 2019; Wilhelm et al., 2013). The so-called state-based framework of working memory, popularised through Cowan's embedded-processes model (Cowan, 1995; Cowan, 1999) and later notably

180 developed by McElree (2006) and Oberauer (2013), is outlined by Adams et al. (2018) as

181 follows:

Information comes in from the environment through a very brief sensory store, activating features in long-term memory corresponding to the sensory properties of the incoming information and its coding: phonological, orthographic, visual, and other in terminology and research focus (e.g., a heightened interest in the role of attention versus modality-specific processing), rather than a clear theoretical break with the earlier multicomponent model that continues to dominate DLD research (Baddeley, 2012). Yet, in

192 our view, the implications of the state-based framework for theory building in DLD are significant. Crucially, the framework encourages a theoretical shift in the locus of impairment

194 from a shortfall in a functionally discrete buffer system (i.e., the phonological loop), to deficits in the quality of long-term speech representations, and the associated efficacy with

196 which such representations become activated in response to features of the speech 
197 environment and are therefore amenable to forming the focus of attention. As Oberauer

198 (2019) has argued, it is essential that long-term representations are encoded in a manner

199 supporting efficient activation and the effective deployment of attention. In this report, we

200 argue that atypical long-term speech representation encoding and activation in DLD result in

201 attention being overloaded in the absence of any fundamental capacity limitation.

202 The challenge for mechanistic accounts arguing that apparent working memory

203 capacity limitations are the consequence of shortfalls in long-term language knowledge is, of

204 course, to explain how and why speech encoding is deficient without appealing to a primary

205 working memory capacity bottleneck. Along these lines, computational modelling of

206 variance in non-word repetition and span task performance among typically developing

207 individuals has appealed to the notions of input frequency and regularity (Jones, 2016; Jones

208 et al., 2007, 2008, 2020; MacDonald \& Christiansen, 2002; Jones et al., 2020; MacDonald \&

209 Christiansen, 2002). Here, the idea is that the ability of an artificial neural network to

210 accurately process any given speech sequence relates directly to the quality of the network's

211 established, analogous representations, which is higher when the relevant input previously

212 received is frequent and structurally consistent. In one landmark study, for instance,

213 MacDonald and Christiansen (2002) showed, in neural networks without functionally discrete

214 working memory systems, that performance deficits analogous to those attributed to verbal

215 working memory capacity limitations by Just and Carpenter (1992) diminished with each

216 cycle of training. This indicates that a separate buffer system which hypothetically varies in

217 capacity between individuals (e.g., a phonological loop) is not required to explain variance in

218 task performance; variance in the frequency of stimulus exposure and therefore the quality of

219 long-term encodings (i.e., more frequently encountered, regular stimuli are better encoded)

220 can parsimoniously account for the data at hand. 
The long-term encoding benefits of high frequency and regularity of exposure clearly

boost performance for certain stimuli in working memory tasks, and may more broadly

explain why working memory capacity appears to increase during infancy and childhood

224 (Jones et al., 2020). Simply, as implicit in the state-based framework of working memory,

task performance may improve as children become increasingly adept at deploying their

mounting long-term language knowledge in the moment, not, as is commonly argued,

because of developmental capacity increases that are independent of the quality of long-term

the degree and quality of language exposure alone is unsatisfactory as an explanation of the language profiles seen in DLD. Evidence for this comes not least from twin studies, which show that dizygotic twins, who are no more genetically similar than regular siblings but largely share a language environment, can be differentially affected by DLD; an observation indicating a genetic component to this disorder (Bishop, 2006). Clearly, then, if we are to better understand how a working memory capacity overload might emerge as a consequence of atypical speech representation, it is necessary to go beyond the notions of input frequency and regularity alone to consider shortfalls in the child's ability to encode speech information from their environment.

Auditory processing deficits commonly reported among children with DLD provide a credible starting point for this form of inquiry. While initially cast as a temporal processing

240 issue, that is, that some children affected by DLD have difficulty discriminating rapidly

241 occurring changes in pure tone - a view developed through the work of Paula Tallal and colleagues (e.g. Merzenich et al., 1996; Tallal et al., 1996) - subsequent studies suggest that

243 the problem may instead lie in frequency discrimination, aside from the speed of stimulus

244 presentation (Bishop et al., 1999; Bishop \& McArthur, 2005; McArthur \& Bishop, 2005a).

245 For instance, in an electroencephalography (EEG) study incorporating an oddball paradigm, 
246 Bishop and McArthur (2005) found group deficits among children with DLD in the ability to

247 identify, through button pressing, differences in frequency between $600 \mathrm{~Hz}$ and $700 \mathrm{~Hz}$ that

248 were independent of the rate of stimulus presentation. Importantly, not only did children with

249 DLD in this study score poorly on behavioural measures (i.e., in their rate of accurate button

250 presses in response to tone sequences), but EEG analysis also highlighted atypical waveforms

251 even when these children made accurate responses. This result suggests that atypical

252 frequency processing may be at play even when performance in a frequency discrimination

253 task, such as those widely used in the initial screening phase of behavioural assessments

254 involving children with DLD, is apparently standard. Frequency discrimination deficits may,

255 therefore, be more widespread than thought in this population.

256 It may appear reasonable to assume a causal association between low-level frequency

257 discrimination deficits and the deficits in higher-order speech representation and retrieval that

258 characterise DLD. Children affected by DLD commonly require more exposures to a spoken

259 word than control children in order to encode similar levels of phonological detail (Gray,

260 2003), for instance, and are often slower and less accurate than age-matched peers when

261 retrieving words and naming known objects (Kambanaros et al., 2015; Messer \& Dockrell,

262 2006), when determining whether an auditory stimulus is a known word or non-word (Jones

263 \& Brandt, 2018), when fixing their gaze to a named visual stimulus (McMurray, Klein-

264 Packard, \& Tomblin., 2019), when identifying words from clipped auditory segments

265 (Montgomery, 1999), when identifying mispronunciations (Alt \& Suddarth, 2012), and, as

266 previously discussed, when repeating non-words (Bishop et al., 1996). These performance

267 deficits between children with and without DLD may be explained in terms of lower

268 familiarity with the target stimuli among children with DLD, which is itself a function of the

269 quality of the speech representations that these children have formed. Evans, Gillam, and

270 Montgomery (2018), for instance, found no spoken word recognition accuracy discrepancies 
271 between children with and without DLD in a gating paradigm in which target word

272 knowledge was controlled. Nevertheless, whether and how such higher-order speech

273 representation deficits relate to underlying abnormalities in frequency discrimination remains

274 unclear, and assuming a casual association here remains controversial in lieu of a satisfactory

275 linking hypothesis (Bishop \& McArthur, 2005; McArthur \& Bishop, 2005).

276 Furthermore, despite a wealth of behavioural evidence pointing to speech

277 representation deficits in children with DLD (e.g. the aforementioned evidence from the

278 naming, mispronunciation identification, and non-word repetition tasks), a precise account of

279 the form that such deficits take remains elusive, with existing research restricted to verbal

280 descriptions of task performance being impeded due to the fuzziness, imprecision, or

281 indistinctiveness of underlying long-term speech representations (Alt \& Suddarth, 2012;

282 Claessen et al., 2009; Claessen \& Leitão, 2012; Maillart et al., 2004). In the current study, we

283 aim to address each of these gaps in current understanding: First, by demonstrating a causal

284 association between auditory processing deficits and deficits in higher-order speech

285 representation and retrieval, and second by providing a precise, computational account of the

286 nature of speech representation and retrieval deficits in DLD that we believe provides an

287 essential counterpart to existing verbal theories. Our aim is to demonstrate how auditory-

288 perceptual deficits can explain deficits in long-term speech representation, which in turn

289 explain communication deficits by way of attention being overloaded, rather than by way of

290 working memory capacity limitations that are independent of the quality of long-term speech

291 encodings.

\section{Speech processing from cochlea to cortex}

The theoretical account presented in this report is informed by the manifold

294 untangling framework developed in visual neuroscience (DiCarlo \& Cox, 2007) and recently

295 applied in studies of speech processing and representation (Kell et al., 2018; Stephenson et 
al., 2020). Manifold untangling describes an integrated theoretical and computational approach to studying neurobiological processes. In this section, our focus is on theory, specifically how manifold untangling shapes the view of speech perception and processing in DLD that we have outlined. Details of the computational implementation of this framework are discussed in the Method section. signals stimulate patterns of firing in populations of neurons that may be understood as a response vector in high dimensional space; a principle illustrated in Figure 1a (Chung et al., 2018; Cohen et al., 2020; DiCarlo et al., 2012; DiCarlo \& Cox, 2007; Stephenson et al., 2020; Yamins \& DiCarlo, 2016). Due to speaker variability, co-articulation effects, and background noise, no two instances of any given spoken word are acoustically identical, and so each spoken instance of a given word stimulates a different neural response vector. The collection of neural response vectors associated with any specific word defines that word's neural manifold.

The manifold untangling framework quantifies changes in the dimensionality and separability of manifolds across a processing hierarchy; in our case the auditory-linguistic pathway (Stephenson et al., 2020). Crucial here is the idea that the manifolds underpinning different spoken words are significantly tangled (i.e., intersecting or overlapping) and thus difficult to separate early in the processing stream (Figure 1b). In the cochlea, for instance,

315 this overlap is due to the responsivity of spiral ganglion cells to low-level acoustic features.

316 Neural representations at this level capture variance in the multiple acoustic signals

317 corresponding to any given spoken word, and are, therefore, described as form dependent or

318 noise sensitive. Transformations instantiated across the typical auditory processing hierarchy

319 result, however, in input-invariant neural responses that are reduced in dimensionality, i.e.,

320 which are substantiated in patterns of activation across relatively small subspaces of a given 
321

322

323

324

325

326

327

328

329

330

331

neural population, and which are therefore more easily separated from the neural response patterns underpinning competitor classes (Figure 1c). In typically developing individuals, this is demonstrated in increasingly form independent or speech selective neural responses across the auditory pathway. Acoustic distortion is shown to stimulate the auditory pathway up to and including at the primary auditory cortex (i.e. the core) and the belt, for instance, with increasing speech selectivity, or, by the same token, reduced sensitivity to low-level acoustic features including noise, then observed in the parabelt and more distal substrates (Davis \& Johnsrude, 2003; DeWitt \& Rauschecker, 2012; Kaas et al., 1999; Okada et al., 2010). This process of transformation defines the central objective of the auditory-linguistic pathway: To establish input-invariant neural speech representations.

The impact of low-level auditory-perceptual deficits on successful manifold untangling (i.e., the shift from form-dependent to form-independent neural responses) is, to our knowledge, as yet unstudied. However, it might be assumed that such auditory-perceptual deficits, which demonstrably characterise the profiles of some children affected by DLD (Bishop \& McArthur, 2005; McArthur \& Bishop, 2005), would prompt atypical trends in neural response transformation throughout the auditory-linguistic pathway. Specifically, we might expect that the degree of untangling achieved on the basis of degraded speech signals would be lower than the degree of untangling achieved on the basis of clean speech signals. Faced with poor auditory processing ability, neural systems may struggle to reduce manifold dimensionality and establish input-invariance, with low-level noise contaminating high-level speech representations and rendering them highly dispersed. The manifold untangling framework therefore has the potential to shape a precise linking hypothesis from low-level auditory-perceptual deficits to higher-order deficits in speech representation in DLD, while providing a formal description of the latter in terms of neural response manifolds characterised by abnormally high dimensionality. 
Furthermore, and fundamental to the primary line of argument pursued in this report,

347 the manifold untangling framework demonstrates how attentional capacity may be

348 overloaded by the low separability of atypically dispersed neural speech representations

349 (Stephenson et al., 2020; Cohen et al., 2020). Recall, for instance, our earlier citation from

350 Oberauer (2019) on the importance of high quality long-term encodings for the effective

351 deployment of attention. Efficient speech recognition and production depend on rapidly and

352 accurately isolating and retrieving required speech representations from an activated long-

353 term memory cohort, a capacity to which attentional control is central. If we assume that

354 auditory-perceptual deficits do characterize the profiles of some children affected by DLD,

355 and if we can show that these low-level deficits are linked to the formation of higher-order

356 speech representations characterised by amplified levels of dispersion and overlap (i.e.,

357 residual manifold tangling), then we might further conclude that the performance profiles

358 commonly attributed to working memory capacity limitations in DLD instead reflect

359 attention being overloaded as a result of long-term speech representations characterised by

360 low discriminability. As we show in the Method section (see Analysis), recent computational

361 realizations of the manifold geometry view of neural responses provide the tools required to

362 formally quantify both speech representation dimensionality and associated demands on

363 attentional capacity (Stephenson et al., 2020; Cohen et al., 2020).

\section{Biological and artificial neural networks}

The purpose of the current study is, then, to demonstrate through computational

366 simulations how working memory capacity deficits may emerge as a consequence of atypical

367 speech representation, which itself results from a primary auditory-perceptual deficit. To do

368 this, we use a deep learning framework involving convolutional neural networks, which we

369 describe further in the Method section (see Model). State-of-the-art deep learning systems

370 have reached human-level accuracy in speech recognition tasks, and work in computational 
371

auditory neuroscience has shown that despite the many substantial differences between biological and artificial neural networks, deep learning can provide valuable insight into human auditory processing and speech representation (e.g. Kell et al., 2018).

There are fundamental parallels between the biological auditory pathway and convolutional network architectures, including the projection of activation into overcomplete space (i.e., activation spreads through layers of an increasing numbers of neurons) and pooling functions (i.e., configurations in which neuron $x$ fires if either antecedent neuron $a, b$, or $c$ fire). The untangling of neural response manifolds is achieved in part as a result of these architectural features, in conjunction with the constraint of response sparseness, i.e., topdown pressure on the system to align on a single target representation. As a result of these constraints, the relative size of the subspace in which manifolds reside decreases at each level of transformation, facilitating manifold separability (DiCarlo \& Cox, 2007; Kell et al., 2018). Nevertheless, closer comparisons of the biological auditory pathway and convolutional neural networks, for instance the position that specific artificial layer activation can predict biological auditory-cortical responses (e.g. Kell et al., 2018) remain controversial (Thompson, 2020). One obvious discrepancy between real-world language processing and the simulations presented in the current report is that natural speech signals unfold in time, while processing in a convolutional neural network does not (Stephenson et al., 2020). For our purposes here, then, networks should be understood as providing computational rather than neurobiological insight, in the tradition of Marr (1982), addressing the following questions: What transformation does speech input undergo in order to achieve spoken word recognition? How is this process of transformation impeded due to a low-level auditory processing deficit? And how does any resultant representational abnormality affect demands on attentional control? 
In the simulations that follow, we model typical and atypical spoken word recognition by presenting deep convolutional neural networks with spectrograms in which information is either retained to mimic typical development or degraded to mimic the auditory processing deficits identified among some children with DLD (Bishop \& McArthur, 2005; McArthur \& Bishop, 2005). Computational simulation is essential in enabling us (i) to examine how speech representation differs in artificial neural systems with and without engineered auditory-perceptual deficits, and (ii) to understand in each case how the form of internal speech representations propagated influences the systems' ability to retrieve any given representation, a capacity understood as central to attentional control. Crucially, in an artificial system, we are able to ensure that any disparities in network performance are not attributable to an input-independent capacity limitation and are instead attributable exclusively to engineered low-level auditory-perceptual deficits. Our models are not intended to provide a complete picture of speech representation and processing deficits in all children affected by DLD. Instead, we aim to detail a specific causal link previously undescribed in the literature, from auditory-perceptual deficits to speech representation deficits to attentional capacity overload, in the absence of any hard-wired capacity limitation.

\section{Method} used to replicate the simulations presented or to experiment with alternative configurations of 414 input, model, and parameters (see https://osf.io/ng6dx/).

\section{$415 \quad$ Model}

416 Simulations involved the ResNet-18 convolutional neural network (He et al., 2015), 417 implemented in Python (Python Software Foundation, 2008) using PyTorch (Paszke et al., 418 2019). A detailed specification of model architecture can be found in the Appendix. For an 419 introduction to convolutional neural networks we recommend Goodfellow et al., (2016; 
https://www.deeplearningbook.org). In essence, in convolutional layers, these networks pass learned filters over the input, here acoustic spectrograms, in order to identify and summarize through pooling functions invariant features that help solve the task at hand, or, more precisely, that help to reduce output and target discrepancy. For instance, the network might learn that identifying a specific formant pattern captured in a certain distribution of pixels facilitates the discrimination of two phonological competitor words (e.g., cat, catch), reducing classification error for these items. We trained and tested two populations of networks $(n=3)$ on clean and degraded speech data in a spoken word recognition task. Training lasted for ten epochs (i.e., full cycles through the training data), determined as the point at which networks exposed to clean input approximated $100 \%$ accuracy in initial trial simulations involving a restricted dataset. capacity between network populations (e.g., number of layers, hidden layer size, or learning rate). As previously described, the current prevailing view is that fundamental working memory capacity limitations cause speech representation and processing deficits among many children affected by DLD. To reflect this position, a prominent approach in the computational modelling of DLD to date has been to reduce network size, particularly the number of nodes in a network's hidden layer, explicitly to mimic group differences in working memory capacity (e.g. Takac et al., 2017; Vitevitch \& Storkel, 2013). In contrast, in the current report, network processing capacity is reconfigured as an emergent rather than a

440 hard-coded, static, and input-independent parameter, with any performance discrepancies 441 observed between network populations attributable only to access to quality low-level 442 acoustic representations.

\section{Data}


Networks were trained and tested on a random sample of 5000 instances of spoken words (4000 training, 1000 test) from the Speech Commands dataset, which comprises .wav files of different articulations of 35 spoken word types used in the development of keyword recognition systems (e.g. backward, up, down, digits 0-9, and a selection of nouns including bird, cat, and dog: see Warden, 2018; see also the Jupyter notebook accompanying the current study). Waveforms were converted to 64-band Mel spectrograms (Stevens et al., 1937), and 0.1 standard deviations of Gaussian noise was added to the training and test data presented to one population of models to simulate the auditory processing deficits observed among some children with DLD (Bishop \& McArthur, 2005). The results of this preprocessing can be seen in Figure 2. Our independent variable is, therefore, dichotomous; either a network has access to high quality auditory information, or it does not. In reality, auditory processing ability is likely to be continuous rather than dichotomous in nature, with DLD describing children at the low end of the distribution (see, for instance, Bishop \& McArthur's, 2005, study of individual differences). Nevertheless, our treatment of auditory processing ability as a dichotomous variable represents a welcome simplifying assumption in this first pass analysis of the role of auditory-perceptual deficits on speech representation and working memory in DLD.

As we noted in our introduction, the existing evidence suggests that the auditoryperceptual deficits seen among some children with DLD are spectral (i.e., frequency based; e.g. Bishop et al., 1999; Bishop \& McArthur, 2005; McArthur \& Bishop, 2005) rather than temporal (e.g. Merzenich et al., 1996; Tallal et al., 1996) in nature. Note, however, that the manner in which we add Gaussian noise to spoken word spectrograms in the current study makes it impossible to distinguish between these contrasting accounts. That is, the addition of noise disrupts both frequency information across the vertical axis and temporal information across the horizontal axis (see Figure 2). This is justified because discriminating between the 
spectral and temporal accounts of auditory processing deficits in DLD is outside of our primary aim to provide an alternative to dominant causal accounts of DLD centred on working memory capacity limitations. With this in mind, we use the general term auditoryperceptual deficit (i.e., instead of frequency processing deficit) throughout the current study.

\section{Analysis}

Networks were required to identify which word each spectrogram corresponded to by outputting a probability distribution over the 35-word lexicon. The word with the highest assigned probability was considered the network's selection. As children with DLD often show word finding deficits and response latencies even when making accurate responses (e.g., Messer \& Dockrell, 2006), we were interested not only in the networks' spectrogram classification accuracy, but also in the degree of certainty in accurate classifications made. This required looking not only at the word with the highest assigned probability, but also at the dispersion or entropy of the predictive distribution output in response to any given spectrogram. High probability, low entropy predictive distributions reflect greater certainty in a prediction and act as proxy for rapid retrieval, while low probability, high entropy predictive distributions reflect the heightened 'consideration' of competitor classes in response to features of the acoustic speech signal presented, and act as proxy for delayed retrieval. probability and entropy are measures of a network's output. However, crucial to the current study was an assessment of the internal speech representations that networks formed. Manifold dimensionality and classification capacity are variables integral to the computational implementation of the manifold untangling framework, and were estimated

492 following the mean-field-theory based method described in Stephenson et al. (2020) across 493 the networks' 20 convolutional layers (see Appendix). Readers interested in the mathematical 
494 principles via which dimensionality and classification capacity are derived are directed to

495 Cohen et al. (2020) and references therein. In essence, dimensionality quantifies the average

496 degree of dispersion in speech representations across a given neural population (i.e., a

497 network layer), while classification capacity quantifies the network's average ability to

498 separate any given internal speech representation from competitor representations in a neural

499 population, and therefore provides a measure of demands on attentional control.

500 Algorithmically, dimensionality and classification capacity are determined by propagating

501 activation through the network in order to determine (i) the embedding dimension of the

502 manifold contributing to successful classification (i.e., dimensionality), and (ii) the number of

503 word representations that can be linearly separated from competitor representations at each

504 level of the network's architecture (i.e., classification capacity), standardizing in each case by

505 layer size in order to account for differences in the number of artificial neurons in each layer

506 (Cohen et al., 2020). High classification capacity indicates neural response manifolds having

507 been reduced in dimensionality to facilitate hyperplane separation (i.e., attention is sufficient;

508 Figure 1c), while low classification capacity indicates high-dimensional manifolds

509 unamenable to efficient hyperplane separation (i.e., attention is overloaded; Figure 1d).

$510 \quad$ Prior research illustrates that dimensionality and classification capacity are not fixed

511 properties (Stephenson et al., 2020). In untrained deep neural networks, little change in

512 manifold dimensionality or classification capacity is seen across layers, from the input layer

513 to the feature layer immediately prior to stimulus classification. In this case, manifolds

514 remain highly dispersed across each layer of the hierarchy, limiting network classification

515 capacity and undermining task performance. However, through training on a specific task,

516 manifold dimensionality decreases across the network hierarchy while classification capacity

517 concurrently increases as a result of improved separability (Chung et al., 2018; Cohen et al.,

518 2020; DiCarlo et al., 2012; DiCarlo \& Cox, 2007; Stephenson et al., 2020; Yamins \& 
519 DiCarlo, 2016). These changes in manifold dimensionality and classification capacity are driven by training and underpin improvements in task performance such as better spoken word classification accuracy.

Through modelling this combination of response variables (i.e., prediction accuracy, probability, and entropy, and manifold dimensionality and classification capacity) as a function input type (i.e., clean versus noisy Mel spectrograms) we were able to analyse both potential variance in performance in a simulated spoken word recognition task and the representation and attentional control factors that underpin that variance. All statistical analyses were conducted in R ( $\mathrm{R}$ Core Team, 2016; see data repository for analysis script).

\section{Results}

Figure 3a shows training error rates by epoch for each network and input type.

530 Networks exposed to clean input showed a spoken word recognition advantage throughout

531 training, with a mean classification accuracy disparity of $79.9 \%(S D=2.21)$ in the clean

532 spectrogram condition, compared to $55.2 \%(S D=1.59)$ in the degraded spectrogram

533 condition. Networks exposed to spectrograms that had been degraded by the addition of

534 Gaussian noise not only made fewer accurate predictions, but also showed substantially greater uncertainty in the accurate predictions they made (Figure 3b, Figure 3c). The entropy of accurate predictive distributions generated by networks exposed to clean input was .18 bits $(S D=.34)$, with a mean, maximum predictive probability of $.94(S D=.13)$. In contrast, networks exposed to degraded input generated accurate predictive distributions with entropy

539 of .53 bits $(S D=.59)$, with a mean maximum predictive probability of $.84(S D=.20)$. These training and test-phase performance profiles relate directly to the networks'

541 ability to represent and efficiently retrieve speech information. In Figure 4, we show the

542 average manifold dimensionality and classification capacity during training at the final

543 convolutional layer of each network, immediately prior to the classification layer (see 
544 Appendix for network specification). Notably, the divergence in manifold dimensionality

545 between networks presented with clean and degraded input was smaller in relatively early

546 training epochs. Through training, each population of networks reduced the average

547 dimensionality of the internal speech representations it formed in this final convolutional

548 layer. Yet, at asymptote, the divergence between network populations was clear: Reducing

549 the dimensionality of degraded input was an obvious challenge for networks simulating speech representation in DLD. These manifold dimensionality reduction deficits are reflected in the complementary analysis of network classification capacity (Figure 4). Classification capacity increased during training across network populations but was substantially higher in networks modelling typical development. This means that the speech representations formed by the networks modelling typical development were discriminated more easily by a simulated attentional control mechanism than the speech representations formed by the networks modelling DLD, in which attentional control was more rapidly exhausted due to excessive processing demands. In essence, the instantiated auditory-perceptual deficit constituted a significant obstacle to learning, resulting in the formation of spoken word representations that were abnormally dispersed and overlapping (i.e., underpinned by common patterns of neural response), and which therefore could not be easily recognised or retrieved.

In Figure 5, a similar trend is shown post training across the networks' 20 convolutional layers. Neural networks exposed to degraded input never reached levels of

564 manifold dimensionality or classification capacity as low as those seen in the layers of the networks exposed to clean input, and these disparities widened substantially towards the final convolutional layer. Again, networks with engineered auditory-perceptual deficits face a greater challenge in reducing speech representation dimensionality, and this directly impedes the ability of these networks to attend to (i.e., isolate and retrieve) specific internal speech 
569 representations. Ultimately, as detailed above, these atypicalities in internal speech representation and simulated attentional control are reflected in disparities in task performance, including reduced speech recognition accuracy and greater uncertainty (i.e.,

572 lower probability, higher entropy predictive distributions) even when accurate classifications 573 are made.

\section{Discussion}

In this article, our aim has been to provide an alternative to dominant causal accounts of DLD centred on working memory capacity limitations. We developed an account of speech perception, representation, and processing in DLD closely aligned with contemporary working memory frameworks that de-emphasise the role of functionally discrete buffer systems such as the phonological loop in exchange for a more parsimonious characterization of working memory in terms of activated long-term memory plus attention (Adams et al., 2018; Chai et al., 2018; Cowan, 1995; D’Esposito \& Postle, 2015; McElree, 2006; Oberauer, 2013, 2019; Wilhelm et al., 2013). We instantiated this theoretical account in a computational model. Simulation demonstrated that protracted manifold untangling provides a plausible link between low-level auditory-perceptual deficits and deficits in higher-order speech representation, as well as a formal description of those speech representation deficits in terms of atypically dispersed patterns of neural response within structures of the auditory-linguistic pathway. This neurocomputational view of speech representation deficits in DLD is broadly consistent with existing verbal descriptions noting the fuzziness, imprecision, or

589 indistinctiveness of these children's speech representations, and provides a vital counterpart 590 to such accounts (Alt \& Suddarth, 2012; Claessen et al., 2009; Claessen \& Leitão, 2012;

591 Maillart et al., 2004). Simulation further illustrated our theoretical view that ostensible shortfalls in working memory capacity may emerge as a consequence of low-level auditory-perceptual deficits 
594 propagating neural response manifolds characterised by atypically high dimensionality and residual tangling. Returning to the trade-off described earlier, this suggests that the challenge facing children with DLD may be one of heightened processing demands rather than one of

597 fixed capacity limitations. Children with DLD may be less able to accurately and rapidly

598 process speech sequences and deploy their long-term language knowledge, whether during

599 listening or production, because that long-term knowledge is poorly configured and not 600 amenable to efficiently forming the focus of attention. We showed that representational 601 atypicality (i.e., the heightened dispersion of artificial neural responses) directly undermined 602 the networks' ability to discriminate any given speech representation within an activated 603 cohort, which is a central function of attentional control. This illustrates how irregularities in 604 long-term speech representation may be the cause of apparent, rather than the consequence of real, working memory capacity shortfalls. Note that this position differs from the claim that atypical auditory processing restricts the maturation of a working memory buffer system

607 that is functionally discrete from long-term language knowledge (e.g., the phonological 608 loop). We posit no such functionally discrete system, and instead attribute a substantial 609 proportion of the variance in working memory task performance to the quality of activated 610 long-term speech encodings. Like prior computational work in this general area (e.g. Jones et 611 al., 2020), the simulations presented here do not provide explicit evidence against a working 612 memory capacity limitation in children with DLD. Rather, they demonstrate a coherent 613 theoretical account of speech perception, representation, and processing deficits in which 614 capacity limitations that are independent of the quality of long-term encodings play no part, 615 and in doing so challenge the status of such limitations as a feature of dominant causal 616 theories of DLD. Simulation also showed how atypical speech representation and control deficits relate 618 not only to reduced performance accuracy in a spoken word recognition task, but also to 
619 substantially greater uncertainty even when making correct responses in that task. Networks

620 with auditory-perceptual deficits made accurate responses characterised by lower maximum

621 probability assignment and higher entropy predictive distributions. This feature of network

622 performance is consistent with behavioural evidence from children with DLD of delays when

623 making accurate responses and associated word finding difficulties, as well as the greater

624 consideration of competitor stimuli in eye-tracking paradigms even when accurate responses

625 are initially made, i.e., a child with DLD first orientates accurately to a visual image

626 corresponding to a presented acoustic label (e.g. net) but subsequently gazes more regularly

627 at competitor images (e.g. a neck) than age-matched, typically developing control children

628 (Kan \& Windsor, 2010; McMurray et al., 2019; Messer \& Dockrell, 2006). Regularly, such

629 patterns of performance have been explained by positing auxiliary, encoding-independent

630 processing constraints, for instance generalised slowing (Kail, 1994) or more specific deficits

631 in a hypothesised lateral inhibition mechanism responsible for the successful dampening of

632 activated long-term competitor representations among typically developing children

633 (McMurray et al., 2019). The modelling work presented in the current study suggests,

634 however, that positing constraints that are independent of the quality of long-term speech

635 representations in order to explain such patterns of performance may be unwarranted.

636 Instead, children's spoken responses may be delayed, or competitor stimuli may be given

637 greater consideration in an eye-tracking paradigm as a result of attention being overloaded by

638 the increased search demands that result from low manifold separability.

639 Above, we commented against drawing close parallels between the convolutional

640 neural networks used in this study and the biological auditory pathway. However, it is

641 notable that the typically developing brain approximates invariant speech-sound

642 representations by the peripheral auditory cortex (Davis \& Johnsrude, 2003), prior to the

643 auditory system splitting into a ventral pathway committed to semantic representation and 
644 processing, and a dorsal pathway committed to speech-segment representation and

645 processing, and articulation; each innervated by frontal neural substrates supporting attention

646 (Hickok \& Poeppel, 2000). This indicates that approximating invariant speech-sound

647 representations at this juncture is essential to the typical function of the language system as a

648 whole, including to ensuring that attentional resources are not exhausted by uneconomical

649 speech encodings. By the same token, this prior work (e.g. Hickok \& Poeppel, 2000) suggests

650 that the protracted manifold untangling simulated in the current report will have wide-

651 reaching implications for the language system as a whole, potentially disrupting the mapping

652 between speech representations and distributed semantics in the ventral stream and speech-

653 segment processing and speech planning in the dorsal stream, as well as disrupting

654 mechanisms of attentional control substantiated in the frontal lobe.

655 Relatedly, it is valuable to note that prior computational work attests to the

656 generalizability of the principles described in this report. While our own focus has been on

657 auditory perception and the encoding of and attention to spoken word representations,

658 previous research strongly suggests that the auditory-perceptual deficits simulated here would

659 prompt protracted manifold untangling regardless of the level of linguistic representation, i.e.,

660 whether phoneme, word, or phrase (Stephenson et al., 2020). Indeed, the principles described

661 here are expected to hold regardless of the modality of the stimuli being classified (e.g.,

662 whether auditory or visual). There is, therefore, nothing special about words as a unit of

663 representation. Across levels of linguistic representation (i.e., phoneme, word, and phrase),

664 speech recognition and comprehension, retrieval, planning, and production would all be

665 expected to be slower and less accurate as a result of attentional capacity being overloaded by

666 high dimensionality impeding the efficient separation of neural response manifolds.

667 Ultimately, determining the coverage of the theory developed here in explaining the broad

668 constellation of deficits seen in DLD is a matter for future research. There is, of course, no 
669 requirement to settle on a single cause of DLD, and indeed such attempts are likely to be 670 fruitless given a complex genetic etiology and the linguistic diversity seen across children 671 with a diagnosis of DLD. Not all children affected by DLD show behavioural deficits or 672 neurophysiological abnormalities in auditory processing (McArthur \& Bishop, 2005), and 673 language impairment is not an inevitable consequence of mild to moderate hearing loss (see 674 Halliday et al., 2017, and references therein). Relatedly, there are features of DLD that are 675 not easily reconciled with the notion of a basis in auditory processing deficits. Hsu and

676 Bishop (2014), for instance, report reliable deficits in the ability of children with DLD to 677 identify regular (though difficult to discern) patterns of change in the position of a character 678 on a computer screen (i.e., in a visual serial reaction time task; though, relatedly, see

679 Marshall et al., 2015, for evidence that nonverbal working memory capacity is impacted by 680 language experience). Thus, the manifold untangling deficit hypothesis described in the 681 current manuscript should be considered a complementary explanatory framework, rather 682 than a unifying or absolute theory of DLD.

683 Attempting to map deficits in manifold untangling to underlying neuronal 684 abnormalities is an important part of the future research agenda. In this report, we situated the 685 locus of deficit at the most fundamental level, the input to the hierarchical processing system. 686 However, given that untangling low-level neural manifolds rests on a protracted and complex 687 hierarchical configuration, including the projection of activation into overcomplete space and 688 pooling functions, it is possible that the problem resides later or more broadly distributed 689 across the auditory pathway, from the basilar membrane to the peripheral auditory cortex, and 690 beyond. Theoretically, unsuccessful manifold untangling may be caused by 691 microneuropathology, in the form of genetic irregularities prompting neuronal mis-migration 692 or inhibiting synaptic pruning, resulting in sub-optimal organisation within the auditory693 linguistic pathway (Bishop, 2014). Future physiological research in this direction might take 
694 lead from work assessing neural responses to distorted speech signals in the auditory cortices

695 of typically developing adults (Davis \& Johnsrude, 2003; DeWitt \& Rauschecker, 2012;

696 Okada et al., 2010). As previously described, this work has identified form-dependent

697 responses to spoken language in the primary auditory cortex and belt, and increasingly form-

698 independent responses in the peripheral auditory cortex and subsequent auditory-linguistic

699 pathways. To our knowledge, it remains unclear whether similar patterns of neural activation

700 across the auditory-linguistic pathway occur in response to different intensities of speech

701 distortion in children with and without DLD.

Given the dominant view that working memory capacity limitations play a causal role in DLD, one line of argument is that interventions specifically targeting working memory can

704 help mitigate these children's language problems (Delage \& Frauenfelder, 2020;

Montgomery et al., 2010). As described in our introduction, a number of commercially available programmes make this claim (e.g., Alloway et al., 2013). There is, however, little empirical evidence supporting the efficacy of working memory training. For instance, in a comprehensive meta-analysis, Melby-Lervåg and Hulme (2013) found no evidence that apparent gains in working memory function either generalized or remained after a delay period. This outcome is fully continuous with the current report, in which one cause of

711 language impairment is considered to be low-level speech perception and encoding deficits,

712 rather than a functionally discrete working memory capacity bottleneck (see also Jones et al.,

713 2020). Collectively, this work casts doubt on the validity of using working memory training

714 as a method of boosting language skills. As an alternative, simulation showed (across training epochs) that increasing the frequency of exposure to specific structures might go some way to

716 improving long-term encoding and, therefore, to improving the accuracy, speed, and

717 confidence with which long-term speech representations are deployed in the moment.

718 Simulation also suggests, however, that increasing frequency of exposure alone is not enough 
to effectively close the gap in representation quality and levels of performance between

children with and without DLD. In Figure 4, we illustrated clear divergence in dimensionality and classification capacity between network populations at asymptote across ten training epochs (a pattern which may differ under longer training regimes). This suggests that more nuanced strategies than simply boosting frequency of exposure are required in order to mitigate the perceptual and representational challenges faced by children affected by DLD.

One such approach, already well-known to clinical practitioners including speech and language therapists, is to control the order of stimulus presentation, for instance by teaching minimal pairs (e.g., cat, catch) in which the discrepant phoneme is a sound that the child has particular difficulties with (Dean et al., 1995). As high-order neural response manifolds adapt to task and communicative demands through time (Stephenson et al., 2020), this approach is expected to improve the discriminability of the representation of the different constituent and therefore the word-level representation. This view re-describes the computational process highlighted in the Method section in which neural networks attune to the specific sub-patterns within speech signals that most effectively reduce performance error. representation during language intervention, here improving spoken word representation (and indeed phrase-level speech representation) by improving sub-lexical speech segment representation. Ultimately, given the complex causal basis of DLD emphasised earlier, comprehensive programmes of intervention that target multiple aspects of the language system appear essential (i.e., because highly specific programs of intervention only focus on remediating a subset of the underlying issues). This factor may explain the limited success of

741 targeted commercial packages of auditory processing intervention such as Fast ForWord

742 (Tallal, 2013) in randomised controlled trials (Strong et al., 2011). Relatedly, it would, as one anonymous reviewer pointed out, be wrong to assume that programs of intervention only 
744 work if they address an identified area of deficit, as working with an area of relative strength

745 may also help overall language functionality. Along these lines, it is reported that individuals

746 with strong semantic (and syntactic) awareness of the language they are perceiving are better

747 able to decode vocoded elements within a sentence by exploiting top-down predictive

748 processing, in the same manner that the occluded orthographic representation $g \# \# d \# n$ might

749 be rapidly decoded by exploiting antecdent information in the phrase "it was a sunny day and

750 the children were playing in the g\#\#d\#n" (i.e. garden; Davis et al., 2005; Sohoglu et al.,

751 2012; see Jones \& Westermann, 2021, for an application of the predictive processing

752 framework to the study of DLD). While it may be challenging to translate this specific

753 research finding directly into a task to use during language intervention, it is nevertheless

754 valuable to note that strengthening semantic and syntactic awareness may help children with

755 DLD navigate the perceptual and representational deficits that constitute a major obstacle to

756 effective communication.

\section{Conclusion}

In this report we have presented an alternative to dominant theoretical accounts of

759 DLD centred on deficits in working memory capacity. Our account aims to reposition the

760 proximal origin of many of the behavioural deficits seen in DLD from a shortfall in working

761 memory capacity, to working memory being itself functionally unimpaired but overloaded

762 due to operating on speech representations characterised by atypically high dimensionality

763 and low separability. 


\section{References}

765

766

767

768

769

770

771

772

773

774

775

776

777

778

779

780

781

782

783

784

785

786

787

Adams, E. J., Nguyen, A. T., \& Cowan, N. (2018). Theories of working memory: Differences in definition, degree of modularity, role of attention, and purpose. Language, Speech, and Hearing Services in Schools, 49(3), 340-355. https://doi.org/10.1044/2018_LSHSS-17-0114

Alloway, T. P., Bibile, V., \& Lau, G. (2013). Computerized working memory training: Can it lead to gains in cognitive skills in students? Computers in Human Behavior, 29(3), 632-638. https://doi.org/10.1016/j.chb.2012.10.023

Alt, M., \& Suddarth, R. (2012). Learning novel words: Detail and vulnerability of initial representations for children with specific language impairment and typically developing peers. Journal of Communication Disorders, 45(2), 84-97. https://doi.org/10.1016/j.jcomdis.2011.12.003

Archibald, L. M. D., \& Gathercole, S. E. (2006a). Short-term and working memory in specific language impairment. International Journal of Language and Communication Disorders, 41(6), 675-693. https://doi.org/10.1080/13682820500442602

Archibald, L. M. D., \& Gathercole, S. E. (2006b). Visuospatial Immediate Memory in Specific Language Impairment. Journal of Speech, Language, and Hearing Research, 49(2), 265-277. https://doi.org/10.1044/1092-4388(2006/022)

Archibald, L. M. D., \& Harder Griebeling, K. (2016). Rethinking the connection between working memory and language impairment. International Journal of Language \& Communication Disorders, 51(3), 252-264. https://doi.org/10.1111/1460-6984.12202

Baddeley, A. (2012). Working memory: Theories, models, and controversies. Annual Review of Psychology, 63(1), 1-29. https://doi.org/10.1146/annurev-psych-120710-100422 
Baddeley, A. D., \& Hitch, G. (1974). Working memory. In G. H. Bower (Ed.), The psychology of learning and motivation: Advances in research and theory (Vol. 8) (pp. 47-89). Academic Press.

Bavin, E. L., Wilson, P. H., Maruff, P., \& Sleeman, F. (2005). Spatio-visual memory of children with specific language impairment: Evidence for generalized processing problems. International Journal of Language \& Communication Disorders, 40(3), 319-332. https://doi.org/10.1080/13682820400027750

Bishop, D. V. M. (2006). What causes specific language impairment in children? Current Directions in Psychological Science, 15(5), 217-221. https://doi.org/10.1111/j.14678721.2006 .00439

Bishop, D. V. M. (2014). Uncommon Understanding (Classic Edition). Psychology Press. https://doi.org/10.4324/9780203381472

Bishop, D. V. M., Bishop, S. J., Bright, P., James, C., Delaney, T., \& Tallal, P. (1999). Different origin of auditory and phonological processing problems in children with language impairment. Journal of Speech, Language, and Hearing Research, 42(1), 155-168. https://doi.org/10.1044/jslhr.4201.155

Bishop, D. V. M., \& McArthur, G. M. (2005). Individual differences in auditory processing in specific language impairment: A follow-up study using event-related potentials and

Bishop, D. V. M., North, T., \& Donlan, C. (1996). Nonword repetition as a behavioural 809 marker for inherited language impairment: Evidence from a twin study. Journal of Child Psychology and Psychiatry, 37(4), 391-403. https://doi.org/10.1111/j.14697610.1996.tb01420.x 
812 Bishop, D. V. M., Snowling, M. J., Thompson, P. A., \& Greenhalgh, T. (2016). CATALISE: A multinational and multidisciplinary delphi consensus study. Identifying language impairments in children. PLOS ONE, 11(7), e0158753. https://doi.org/10.1371/journal.pone.0158753

Boerma, T., \& Blom, E. (2020). Effects of developmental language disorder and bilingualism on children's executive functioning: A longitudinal study. Research in Developmental Disabilities, 107, 103782. https://doi.org/10.1016/j.ridd.2020.103782

Chai, W. J., Abd Hamid, A. I., \& Abdullah, J. M. (2018). Working memory from the psychological and neurosciences perspectives: A review. Frontiers in Psychology, 9. https://doi.org/10.3389/fpsyg.2018.00401

Chung, S., Lee, D. D., \& Sompolinsky, H. (2018). Classification and Geometry of General Perceptual Manifolds. Physical Review X, 8(3), 31003. https://doi.org/10.1103/PhysRevX.8.031003

Claessen, M., Heath, S., Fletcher, J., Hogben, J., \& Leitão, S. (2009). Quality of phonological representations: A window into the lexicon? International Journal of Language and Communication Disorders, 44(2), 121-144. https://doi.org/10.1080/13682820801966317

Claessen, M., \& Leitão, S. (2012). Phonological representations in children with SLI. Child Language Teaching and Therapy, 28(2), 211-223. https://doi.org/10.1177/0265659012436851

832 Coady, J. A., \& Evans, J. L. (2008). Uses and interpretations of non-word repetition tasks in 833 children with and without specific language impairments (SLI). International Journal of Language and Communication Disorders, 43(1), 1-40. https://doi.org/10.1080/13682820601116485 
836 Cohen, U., Chung, S. Y., Lee, D. D., \& Sompolinsky, H. (2020). Separability and geometry of object manifolds in deep neural networks. Nature Communications, 11(1), 1-13. https://doi.org/10.1038/s41467-020-14578-5

Cowan, N. (1995). Attention and Memory: An Integrated Framework. Oxford University Press.

841 Working Memory (pp. 62-101). Cambridge University Press. https://doi.org/10.1017/CBO9781139174909.006

Davis, M. H., \& Johnsrude, I. S. (2003). Hierarchical processing in spoken language comprehension. Journal of Neuroscience, 23(8), 3423-3431. https://doi.org/10.1523/jneurosci.23-08-03423.2003

Davis, M. H., Johnsrude, I. S., Hervais-Adelman, A., Taylor, K., \& McGettigan, C. (2005). Lexical information drives perceptual learning of distorted speech: Evidence from the comprehension of noise-vocoded sentences. Journal of Experimental Psychology: General, 134(2), 222-241. https://doi.org/10.1037/0096-3445.134.2.222

Dean, E. C., Howell, J., Waters, D., \& Reid, J. (1995). Metaphon: A metalinguistic approach to the treatment of phonological disorder in children. Clinical Linguistics \& Phonetics, 9(1), 1-19. https://doi.org/10.3109/02699209508985318

Delage, H., \& Durrleman, S. (2018). Developmental dyslexia and specific language impairment: Distinct syntactic profiles? Clinical Linguistics \& Phonetics, 32(8), 758785. https://doi.org/10.1080/02699206.2018.1437222

Delage, H., \& Frauenfelder, U. H. (2020). Relationship between working memory and complex syntax in children with Developmental Language Disorder. Journal of Child Language, 47(3), 600-632. https://doi.org/10.1017/S0305000919000722 
860 D’Esposito, M., \& Postle, B. R. (2015). The Cognitive Neuroscience of Working Memory.

861

862

863

864

865

866

867

868

869

870

871

872

873

874

875

876

877

878

879

880

881

882

883

884 Annual Review of Psychology, 66(1), 115-142. https://doi.org/10.1146/annurevpsych-010814-015031

DeWitt, I., \& Rauschecker, J. P. (2012). Phoneme and word recognition in the auditory ventral stream. Proceedings of the National Academy of Sciences of the United States of America, 109(8), 505-514. https://doi.org/10.1073/pnas.1113427109

DiCarlo, J. J., \& Cox, D. D. (2007). Untangling invariant object recognition. Trends in Cognitive Sciences, 11(8), 333-341. https://doi.org/10.1016/j.tics.2007.06.010

DiCarlo, J. J., Zoccolan, D., \& Rust, N. C. (2012). How does the brain solve visual object recognition? Neuron, 73(3), 415-434. https://doi.org/10.1016/j.neuron.2012.01.010

Durrleman, S., \& Delage, H. (2016). Autism spectrum disorder and specific language impairment: Overlaps in syntactic profiles. Language Acquisition, 23(4), 361-386. https://doi.org/10.1080/10489223.2016.1179741

Ellis Weismer, S., Davidson, M. M., Gangopadhyay, I., Sindberg, H., Roebuck, H., \& Kaushanskaya, M. (2017). The role of nonverbal working memory in morphosyntactic processing by children with specific language impairment and autism spectrum disorders. Journal of Neurodevelopmental Disorders, 9(1), 28. https://doi.org/10.1186/s11689-017-9209-6

Evans, J. L., Gillam, R. B., \& Montgomery, J. W. (2018). Cognitive Predictors of Spoken Word Recognition in Children With and Without Developmental Language Disorders. Journal of Speech, Language, and Hearing Research, 61(6), 1409-1425. https://doi.org/10.1044/2018_JSLHR-L-17-0150

Gathercole, S. E. (1995). Is nonword repetition a test of phonological memory or long-term knowledge? It all depends on the nonwords. Memory \& Cognition, 23(1), 83-94. https://doi.org/10.3758/BF03210559 
885 Gathercole, S. E., Pickering, S. J., Ambridge, B., \& Wearing, H. (2004). The structure of 886 working memory from 4 to 15 years of age. Developmental Psychology, 40(2), 177190. https://doi.org/10.1037/0012-1649.40.2.177

\section{8}

Goodfellow, I., Bengio, Y., \& Courville, A. (2016). Deep Learning. MIT Press.

Gray, S. (2003). Word-Learning by Preschoolers With Specific Language Impairment. Journal of Speech Language and Hearing Research, 46(1), 56. https://doi.org/10.1044/1092-4388(2003/005)

Halliday, L. F., Tuomainen, O., \& Rosen, S. (2017). Language Development and Impairment in Children With Mild to Moderate Sensorineural Hearing Loss. Journal of Speech, Language, and Hearing Research, 60(6), 1551-1567. https://doi.org/10.1044/2016_JSLHR-L-16-0297

He, K., Zhang, X., Ren, S., \& Sun, J. (2015). Deep residual learning for image recognition. Henry, L. A., \& Botting, N. (2017). Working memory and developmental language impairments. Child Language Teaching and Therapy, 33(1), 19-32. https://doi.org/10.1177/0265659016655378

Hickok, G., \& Poeppel, D. (2000). Towards a functional neuroanatomy of speech perception. Trends in Cognitive Sciences, 4(4), 131-138. https://doi.org/10.1016/S13646613(00)01463-7

Howard, D., \& Lely, H. K. J. van der. (1995). Specific language impairment in children is not due to a short-term memory deficit: Response to Gathercole \& Baddeley. Journal of Speech, Language, and Hearing Research, 38(2), 466-472. https://doi.org/10.1044/jshr.3802.466 
910 Hsu, H. J., \& Bishop, D. V. M. (2014). Sequence-specific procedural learning deficits in

911 children with specific language impairment. Developmental Science, 17(3), 352-365.

912 https://doi.org/10.1111/desc. 12125

913 Jakubowicz, C. (2011). Measuring derivational complexity: New evidence from typically

914 developing and SLI learners of L1 French. Lingua, 121(3), 339-351.

915 https://doi.org/10.1016/j.lingua.2010.10.006

916 Jones, G. (2016). The influence of children's exposure to language from two to six years: The

917 case of nonword repetition. Cognition, 153, 79-88.

918 https://doi.org/10.1016/j.cognition.2016.04.017

919 Jones, G., Gobet, F., \& Pine, J. (2008). Computer simulations of developmental change: The

920 contributions of working memory capacity and long-term knowledge. Cognitive

921 Science: A Multidisciplinary Journal, 32(7), 1148-1176.

922 https://doi.org/10.1080/03640210802073689

923 Jones, G., Gobet, F., \& Pine, J. M. (2007). Linking working memory and long-term memory: 924 A computational model of the learning of new words. Developmental Science, 10(6), 925 853-873. https://doi.org/10.1111/j.1467-7687.2007.00638.x

926 Jones, G., Justice, L. V., Cabiddu, F., Lee, B. J., Iao, L.-S., Harrison, N., \& Macken, B.

927 (2020). Does short-term memory develop? Cognition, 198, 104200.

928 https://doi.org/10.1016/j.cognition.2020.104200

929 Jones, S. D., \& Westermann, G. (2021, May 25). Under-resourced or overloaded? Rethinking 930 working memory deficits in developmental language disorder. OSF Preprints.

931 https://doi.org/10.31219/osf.io/rb5jf

932 Jones, S. D., \& Brandt, S. (2018). Auditory lexical decisions in developmental language disorder: A meta-analysis of behavioral studies. Journal of Speech, Language, and 

0447

Jones, S. D., \& Westermann, G. (2021). Predictive processing and developmental language disorder. Journal of Speech, Language, and Hearing Research, 64(1), 181-185.

938 https://doi.org/10.1044/2020_JSLHR-20-00409

Just, M. A., \& Carpenter, P. A. (1992). A capacity theory of comprehension: Individual differences in working memory. Psychological Review, 99(1), 122-149. https://doi.org/10.1037/0033-295X.99.1.122

Kaas, J. H., Hackett, T. A., \& Tramo, M. J. (1999). Auditory processing in primate cerebral cortex. Current Opinion in Neurobiology, 9(2), 164-170. https://doi.org/10.1016/S0959-4388(99)80022-1

Kail, R. (1994). A method for studying the generalized slowing hypothesis in children with 946 specific language impairment. Journal of Speech, Language, and Hearing Research, 37(2), 418-421. https://doi.org/10.1044/jshr.3702.418

Kalnak, N., Peyrard-Janvid, M., Forssberg, H., \& Sahlén, B. (2014). Nonword Repetition - A

950 clinical marker for specific language impairment in swedish associated with parents' language-related problems. PLoS ONE, 9(2), e89544. https://doi.org/10.1371/journal.pone.0089544

Kambanaros, M., Michaelides, M., \& Grohmann, K. K. (2015). Measuring word retrieval deficits in a multilingual child with SLI: Is there a better language? Journal of Neurolinguistics, 34, 112-130. https://doi.org/10.1016/j.jneuroling.2014.09.006

Kan, P. F., \& Windsor, J. (2010). Word Learning in Children With Primary Language Impairment: A Meta-Analysis. Journal of Speech, Language, and Hearing Research, 53(3), 739-756. https://doi.org/10.1044/1092-4388(2009/08-0248) 
Kell, A. J. E., Yamins, D. L. K., Shook, E. N., Norman-Haignere, S. V., \& McDermott, J. H. (2018). A task-optimized neural network replicates human auditory behavior, predicts brain responses, and reveals a cortical processing hierarchy. Neuron, 98(3), 630644.e16. https://doi.org/10.1016/j.neuron.2018.03.044

Kluyver, T., Ragan-Kelley, B., Pérez, F., Granger, B., Bussonnier, M., Frederic, J., Kelley, K., Hamrick, J., Grout, J., Corlay, S., Ivanov, P., Avila, D., Abdalla, S., \& Willing, C. (2016). Jupyter Notebooks - A publishing format for reproducible computational workflows. Positioning and Power in Academic Publishing: Players, Agents and Agendas - Proceedings of the 20th International Conference on Electronic Publishing, ELPUB 2016, 87-90. https://doi.org/10.3233/978-1-61499-649-1-87

MacDonald, M. C., \& Christiansen, M. H. (2002). Reassessing working memory: Comment on Just and Carpenter (1992) and Waters and Caplan (1996). Psychological Review, 109(1), 35-54. https://doi.org/10.1037/0033-295X.109.1.35

Maillart, C., Schelstraete, M.-A., \& Hupet, M. (2004). Phonological representations in children with SLI: A study of French. Journal of Speech, Language, and Hearing Research, 47(1), 187-198. https://doi.org/10.1044/1092-4388(2004/016)

Marr, D. (1982). Vision: A Computational Investigation into the Human Representation and Processing of Visual Information. Henry Holt and Co., Inc.

Marrone, N., Alt, M., DeDe, G., Olson, S., \& Shehorn, J. (2015). Effects of steady-state noise on verbal working memory in young adults. Journal of Speech, Language, and Hearing Research, 58(6), 1793-1804. https://doi.org/10.1044/2015_JSLHR-H-140223

Marshall, C., Jones, A., Denmark, T., Mason, K., Atkinson, J., Botting, N., \& Morgan, G. (2015). Deaf children's non-verbal working memory is impacted by their language experience. Frontiers in Psychology, 6. https://doi.org/10.3389/fpsyg.2015.00527 
983

984

985

986

987

988

989

990

991

992

993

994

995

996

997

998

999

1000

1001

1002

1003

1004

1005

1006

1007

McArthur, G. M., \& Bishop, D. V. M. (2005a). Speech and non-speech processing in people with specific language impairment: A behavioural and electrophysiological study. Brain and Language, 94(3), 260-273. https://doi.org/10.1016/j.band1.2005.01.002

McArthur, G. M., \& Bishop, D. V. M. (2005b). Speech and non-speech processing in people with specific language impairment: A behavioural and electrophysiological study. Brain and Language, 94(3), 260-273. https://doi.org/10.1016/j.band1.2005.01.002

McElree, B. (2006). Accessing recent events (pp. 155-200). https://doi.org/10.1016/S00797421(06)46005-9

McMurray, B., Klein-Packard, J., \& Tomblin, J. B. (2019). A real-time mechanism underlying lexical deficits in developmental language disorder: Between-word inhibition. Cognition, 191, 104000. https://doi.org/10.1016/j.cognition.2019.06.012

Melby-Lervåg, M., \& Hulme, C. (2013). Is working memory training effective? A metaanalytic review. Developmental Psychology, 49(2), 270-291. https://doi.org/10.1037/a0028228

Melby-Lervåg, M., Lervåg, A., Lyster, S.-A. H., Klem, M., Hagtvet, B., \& Hulme, C. (2012). Nonword-repetition ability does not appear to be a causal influence on children's vocabulary development. Psychological Science, 23(10), 1092-1098. https://doi.org/10.1177/0956797612443833

Merzenich, M. M., Jenkins, W. M., Johnston, P., Schreiner, C., Miller, S. L., \& Tallal, P. (1996). Temporal Processing Deficits of Language-Learning Impaired Children Ameliorated by Training. Science, 271(5245), 77-81. https://doi.org/10.1126/science.271.5245.77

Messer, D., \& Dockrell, J. E. (2006). Children's naming and word-finding difficulties: Descriptions and explanations. Journal of Speech, Language, and Hearing Research, 49(2), 309-324. https://doi.org/10.1044/1092-4388(2006/025) 
1008

1009

1010

1011

1012

1013

1014

1015

1016

1017

1018

1019

1020

1021

1022

1023

1024

1025

1026

1027

1028

1029

1030

Montgomery, J. W. (1995). Sentence comprehension in children with specific language impairment: The role of phonological working memory. Journal of Speech, Language, and Hearing Research, 38(1), 187-199. https://doi.org/10.1044/jshr.3801.187

Montgomery, J. W. (1999). Recognition of gated words by children with specific language impairment: An examination of lexical mapping. Journal of Speech, Language, and Hearing Research, 43(3), 735-743.

Montgomery, J. W. (2003). Working memory and comprehension in children with specific language impairment: What we know so far. Journal of Communication Disorders, 36(3), 221-231. https://doi.org/10.1016/S0021-9924(03)00021-2

Montgomery, J. W., Gillam, R. B., Evans, J. L., Schwartz, S., \& Fargo, J. D. (2019). A comparison of the storage-only deficit and joint mechanism deficit hypotheses of the verbal working memory storage capacity limitation of children with developmental language disorder. Journal of Speech, Language, and Hearing Research, 62(10), 3808-3825. https://doi.org/10.1044/2019_JSLHR-L-19-0071

Montgomery, J. W., Magimairaj, B. M., \& Finney, M. C. (2010). Working memory and specific language impairment: An update on the relation and perspectives on assessment and treatment. American Journal of Speech-Language Pathology, 19(1), 78-94. https://doi.org/10.1044/1058-0360(2009/09-0028)

Norbury, C. F., Gooch, D., Wray, C., Baird, G., Charman, T., Simonoff, E., Vamvakas, G., \& Pickles, A. (2016). The impact of nonverbal ability on prevalence and clinical presentation of language disorder: Evidence from a population study. Journal of Child Psychology and Psychiatry, 57(11), 1247-1257. https://doi.org/10.1111/jcpp.12573 
1031 Oberauer, K. (2013). The focus of attention in working memory-From metaphors to mechanisms. Frontiers in Human Neuroscience, 7. https://doi.org/10.3389/fnhum.2013.00673

Oberauer, K. (2019). Working memory and attention - A conceptual analysis and review. Journal of Cognition, 2(1). https://doi.org/10.5334/joc.58

Okada, K., Rong, F., Venezia, J., Matchin, W., Hsieh, I. H., Saberi, K., Serences, J. T., \& Hickok, G. (2010). Hierarchical organization of human auditory cortex: Evidence from acoustic invariance in the response to intelligible speech. Cerebral Cortex, 20(10), 2486-2495. https://doi.org/10.1093/cercor/bhp318

Paszke, A., Gross, S., Massa, F., Lerer, A., Bradbury, J., Chanan, G., Killeen, T., Lin, Z., Gimelshein, N., Antiga, L., Desmaison, A., Köpf, A., Yang, E., DeVito, Z., Raison, M., Tejani, A., Chilamkurthy, S., Steiner, B., Fang, L., ... Chintala, S. (2019). PyTorch: An imperative style, high-performance deep learning library.

Python Software Foundation. (2008). Python. In Python Language Reference (No. 3). https://www.python.org/

R Core Team. (2016). R. In $R$ Core Team.

Sohoglu, E., Peelle, J. E., Carlyon, R. P., \& Davis, M. H. (2012). Predictive top-down integration of prior knowledge during speech perception. Journal of Neuroscience,

1050 Stephenson, C., Feather, J., Padhy, S., Elibol, O., Tang, H., McDermott, J., \& Chung, S. (2020). Untangling in Invariant Speech Recognition.

Stevens, S. S., Volkmann, J., \& Newman, E. B. (1937). A scale for the measurement of the psychological magnitude pitch. The Journal of the Acoustical Society of America, 8(3), 185-190. https://doi.org/10.1121/1.1915893 
1055

1056

1057

1058

1059

1060

1061

1062

1063

1064

1065

1066

1067

1068

1069

1070

1071

1072

1073

1074

1075

1076

1077

1078

1079

Strong, G. K., Torgerson, C. J., Torgerson, D., \& Hulme, C. (2011). A systematic metaanalytic review of evidence for the effectiveness of the 'Fast ForWord' language intervention program. Journal of Child Psychology and Psychiatry, 52(3), 224-235. https://doi.org/10.1111/j.1469-7610.2010.02329.x

Takac, M., Knott, A., \& Stokes, S. F. (2017). What can Neighbourhood Density effects tell us about word learning? Insights from a connectionist model of vocabulary development. Journal of Child Language, 44(2), 346-379.

https://doi.org/10.1017/S0305000916000052

Tallal, P. (2013). Fast ForWord®. In Progress in Brain Research (Vol. 207, pp. 175-207). Elsevier. https://doi.org/10.1016/B978-0-444-63327-9.00006-0

Tallal, P., Miller, S. L., Bedi, G., Byma, G., Wang, X., Nagarajan, S. S., Schreiner, C., Jenkins, W. M., \& Merzenich, M. M. (1996). Language Comprehension in LanguageLearning Impaired Children Improved with Acoustically Modified Speech. Science, 271(5245), 81-84. https://doi.org/10.1126/science.271.5245.81

Thompson, J. A. F. (2020). Characterizing and comparing acoustic representations in convolutional neural networks and the human auditory system. Université de Montréal.

Van Bon, W. H. J., \& Van Der Pijl, J. M. L. (1997). Effects of word length and wordlikeness on pseudoword repetition by poor and normal readers. Applied Psycholinguistics, 18(1), 101-114. https://doi.org/10.1017/S0142716400009899

Van Der Lely, H. K. J., \& Howard, D. (1993). Children with specific language impairment: Linguistic impairment or short-term memory deficit? Journal of Speech, Language, and Hearing Research, 36(6), 1193-1207. https://doi.org/10.1044/jshr.3606.1193

Vance, M. (2008). Short-term memory in children with developmental language disorder. In C. F. Norbury, J. B. Tomblin, \& D. V. M. Bishop (Eds.), Understanding 
1080

1081

1082

1083

1084

1085

1086

1087

1088

1089

1090

1091

1092

1093

1094

1095 Developmental Language Disorders: From Theory to Practice (pp. 23-38). Psychology Press.

Vitevitch, M. S., \& Storkel, H. L. (2013). Examining the Acquisition of Phonological Word Forms with Computational Experiments. Language and Speech, 56(4), 493-527. https://doi.org/10.1177/0023830912460513

Warden, P. (2018). Speech commands: A dataset for limited-vocabulary speech recognition.

Wilhelm, O., Hildebrandt, A., \& Oberauer, K. (2013). What is working memory capacity, and how can we measure it? Frontiers in Psychology, 4. https://doi.org/10.3389/fpsyg.2013.00433

Yamins, D. L. K., \& DiCarlo, J. J. (2016). Using goal-driven deep learning models to understand sensory cortex. Nature Neuroscience, 19(3), 356-365. https://doi.org/10.1038/nn.4244

Zebib, R., Tuller, L., Hamann, C., Abed Ibrahim, L., \& Prévost, P. (2020). Syntactic complexity and verbal working memory in bilingual children with and without Developmental Language Disorder. First Language, 40(4), 461-484. https://doi.org/10.1177/0142723719888372 
1097 Figure 1

1098 Illustration of manifold untangling across the auditory and language pathways in typical

1099 development and DLD

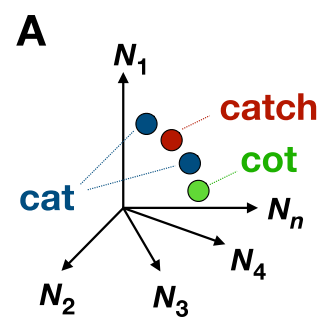

B
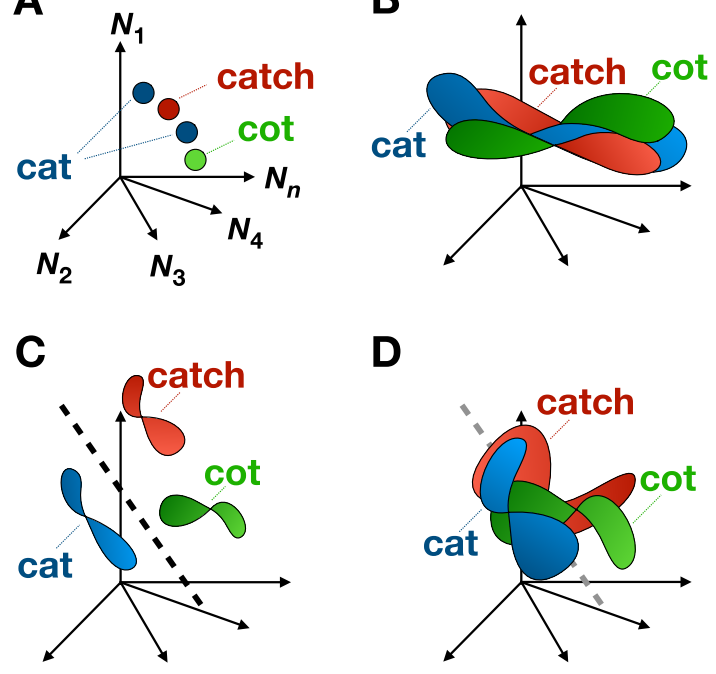

1100

Note. (A) the spoken words cat, catch, and cot in high dimensional space, with each axis $\left(N_{1}\right.$

to $N_{\mathrm{n}}$ ) illustrating the response of a single neuron in a population, in spikes per second. Two

1103 spoken instances of the same word, e.g., cat, will reside in a different neural response vector.

1104 (B) collectively, response vectors associated with any given word form a manifold.

1105 Manifolds of different words are tangled early in the auditory-linguistic pathway due to

1106 cellular responsiveness to low-level acoustic features. (C; a high-capacity system) manifolds

1107 are incrementally untangled throughout the auditory pathway, eventually supporting efficient

1108 discrimination and reducing attentional demand. (D; a low-capacity system) in DLD, a low-

1109 level auditory-perceptual deficit may mean that manifold untangling is protracted, leading to

1110 abnormally high-dimensional, high-order speech representations that are more difficult to

1111 discriminate and which therefore overwhelm attentional capacity. 


\section{$1112 \quad$ Figure 2}

1113 Mel spectrograms of the word 'backward', clean and with Gaussian noise (SD = 0.1)

1114
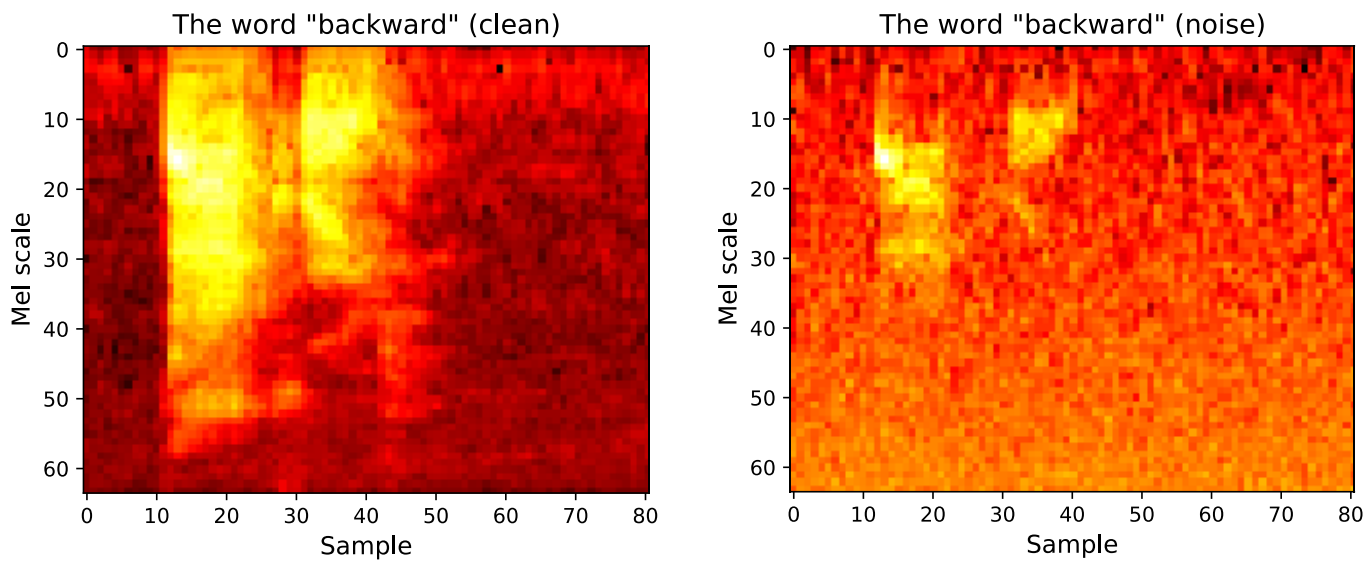


\section{Figure 3}

1116 Network performance during training and testing

A

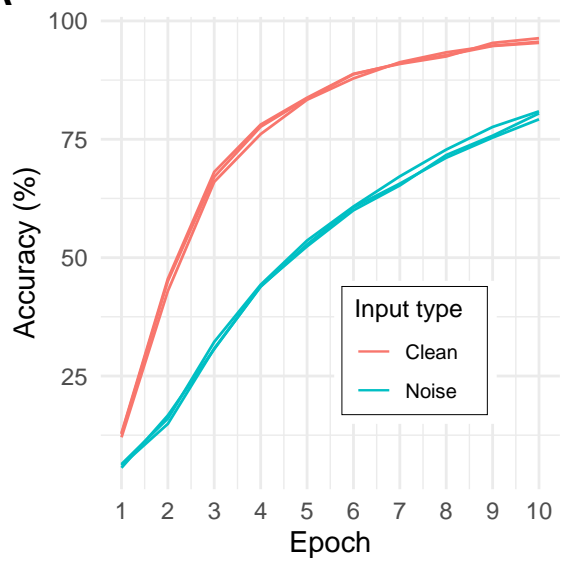

B


1117

1118 Note. (A) accuracy (\%) by training epoch and input type. (B) accurate response predictive

1119 distribution entropy in bits as a function of input type. (C) probability assigned to accurate

1120 predictions as a function of input type. In (B) and (C) black dots represent raw data points,

1121 filled portions illustrate densities, and black horizonal bars illustrate means. 


\section{Figure 4}

1123 Feature layer dimensionality and classification capacity by input type and training epoch
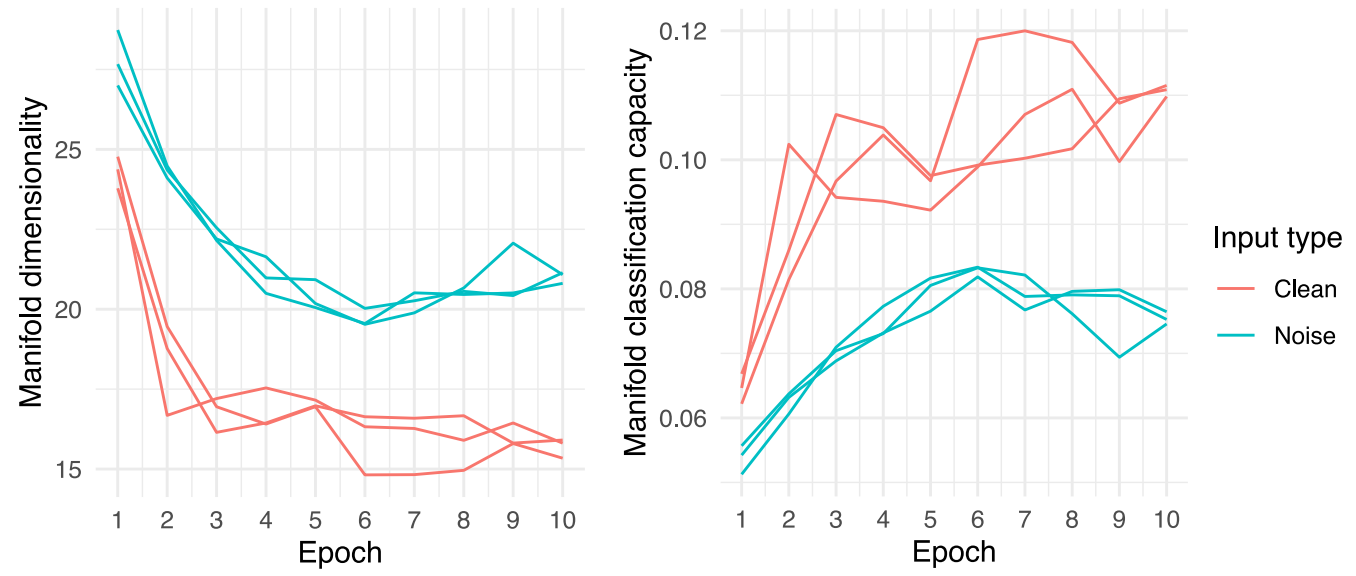


\section{$1125 \quad$ Figure 5}

1126 Post-training dimensionality and classification capacity by convolutional layer and input

1127 type
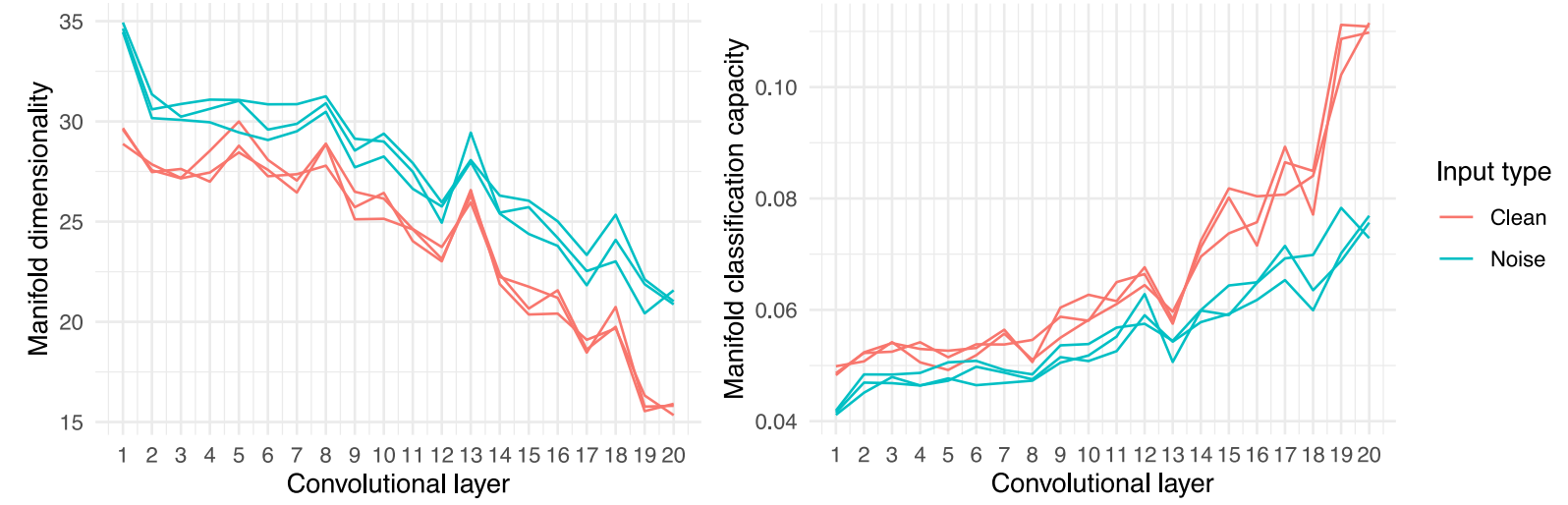


\section{Appendix}

1130

\section{ResNet-18 specification}

\begin{tabular}{|c|c|c|c|c|}
\hline Layer index & Layer name & Output size & Kernel size & Stride \\
\hline 1 & Conv. 2D & 1,64 & 7,7 & 2,2 \\
\hline 2 & Conv. 2D & 64,64 & 3,3 & 1,1 \\
\hline 3 & Conv. 2D & 64,64 & 3,3 & 1,1 \\
\hline 4 & Conv. 2D & 64,64 & 3,3 & 1,1 \\
\hline 5 & Conv. 2D & 64,64 & 3,3 & 1,1 \\
\hline 6 & Conv. 2D & 64,128 & 3,3 & 2,2 \\
\hline 7 & Conv. 2D & 128,128 & 3,3 & 1,1 \\
\hline 8 & Conv. 2D & 64,128 & 1,1 & 2,2 \\
\hline 9 & Conv. 2D & 128,128 & 3,3 & 1,1 \\
\hline 10 & Conv. 2D & 128,128 & 3,3 & 1,1 \\
\hline 11 & Conv. 2D & 128,256 & 3,3 & 2,2 \\
\hline 12 & Conv. 2D & 256,256 & 3,3 & 1,1 \\
\hline 13 & Conv. 2D & 128,256 & 1,1 & 2,2 \\
\hline 14 & Conv. 2D & 256,256 & 3,3 & 1,1 \\
\hline 15 & Conv. 2D & 256,256 & 3,3 & 1,1 \\
\hline 16 & Conv. 2D & 256,512 & 3,3 & 2,2 \\
\hline 17 & Conv. 2D & 512,512 & 3,3 & 1,1 \\
\hline 18 & Conv. 2D & 256,512 & 1,1 & 2,2 \\
\hline 19 & Conv. 2D & 512,512 & 3,3 & 1,1 \\
\hline 20 & Conv. 2D & 512,512 & 3,3 & 1,1 \\
\hline 21 & Linear & 35 & $\mathrm{n} / \mathrm{a}$ & $\mathrm{n} / \mathrm{a}$ \\
\hline
\end{tabular}

1131

Hyperparameters

Optimizer: stochastic gradient descent

Learning rate: 001

Momentum: .9

Loss function: cross-entropy loss

1133 Note. See Jupyter Notebook for activation functions and pooling, normalisation, and dropout 1134 layers. 